\title{
Holographic Lifshitz fermions and exponentially suppressed spectral weight
}

\author{
Youngshin $\mathrm{Kim}^{a, b}$ and James T. Liu ${ }^{b}$ \\ ${ }^{a}$ C.N. Yang Institute for Theoretical Physics, Department of Physics and Astronomy, \\ Stony Brook University, \\ Stony Brook, NY 11794-3840, U.S.A. \\ ${ }^{b}$ Michigan Center for Theoretical Physics, Randall Laboratory of Physics, \\ The University of Michigan, \\ Ann Arbor, MI 48109-1040, U.S.A. \\ E-mail: youngshin.kim.1@stonybrook.edu, jimliu@umich.edu
}

ABSTRACT: The absence of fixed momentum excitations in a theory with Lifshitz scale invariance gives rise to exponential suppression of spectral weight in the low-frequency limit. In the holographic dual, this suppression arises as a consequence of a tunneling barrier that decouples the horizon from the boundary. We compute the spin- $1 / 2$ holographic Green's function and show that the form of the barrier is identical to that of the scalar case. We furthermore demonstrate that the suppression factor is universal in the $\hat{\omega} \rightarrow 0$ limit where $\hat{\omega}=\omega /|\vec{k}|^{z}$. In particular, it depends only on $\hat{\omega}$ and the critical exponent $z$, and is independent of scaling dimension and spin.

KEYwords: AdS-CFT Correspondence, Holography and condensed matter physics (AdS/CMT)

ARXIV EPRINT: 1603.06959 


\section{Contents}

1 Introduction $\quad 1$

2 The Dirac equation in a Lifshitz background 3

2.1 Extracting the holographic Green's function 5

3 The holographic Green's function in the WKB approximation $\quad 7$

3.1 Exponential suppression in the $\hat{\omega} \rightarrow 0$ limit 9

$\begin{array}{lll}4 & \text { Discussion } & 10\end{array}$

A The WKB approximation for $1 / r^{2}$ potentials $\quad 12$

$\begin{array}{ll}\text { A.1 Connection formulae } & 13\end{array}$

A.2 Handling the singular $1 / r^{2}$ potential 14

B The Green's function from the numerical method $\quad 15$

\section{Introduction}

Following the remarkable successes of AdS/CFT and its applications to strongly coupled gauge theories, there has been much recent interest in developing a similar program for nonrelativistic duals. In particular, quantum critical phenomena with the scaling symmetry

$$
t \rightarrow \lambda^{z} t, \quad \vec{x} \rightarrow \lambda \vec{x}
$$

where $z$ is the dynamical exponent, can be described in the framework of Lifshitz holography with a bulk geometry given by [1]

$$
d s_{n+2}^{2}=-\left(\frac{L}{r}\right)^{2 z} d t^{2}+\left(\frac{L}{r}\right)^{2}\left(d \vec{x}_{n}^{2}+d r^{2}\right) .
$$

Here we have chosen the radial coordinate so that the boundary scaling (1.1) is associated with the transformation $r \rightarrow \lambda r$.

Much of the analysis of Lifshitz holography follows from the well-developed methods of relativistic AdS/CFT. However, there are some key differences. Firstly, while the bulk metric (1.2) may in some sense be viewed as a generalization of the Poincaré patch of AdS, it in fact has a tidal singularity at the horizon [1-4]. There are of course various approaches to handling this mild singularity [5-8]. However, a more substantial difference appears in the causal structure of the Lifshitz spacetime. In particular, while null geodesics in an AdS background will reach the boundary in finite coordinate time, only radial null geodesics will 
do so in a Lifshitz background [9]. Thus, in the classical limit, boundary probes carrying transverse momentum decouple from the interior of the Lifshitz spacetime.

It was demonstrated in [9] that this decoupling persists at the quantum level in that bulk scalar modes carrying large transverse momentum become 'trapped' at the horizon in the sense that they leave only an exponentially small imprint on the boundary. As a consequence, the smearing function that maps from the boundary to the bulk is ill-defined in a strict sense, and can at most be viewed only as a distribution. Moreover, this feature of Lifshitz holography remains even when the tidal singularity at the horizon is removed.

The origin of the trapped scalar modes can be seen by investigating the effective potential for the radial part of the Klein-Gordon equation. In contrast with a pure AdS background, the Lifshitz geometry gives rise to a tunneling barrier for sufficiently large spatial momentum. This in turn leads to exponential suppression in the radial wavefunction that decouples the corresponding mode from the boundary. This suppression also shows up in the holographic Green's function in the form of an exponentially suppressed spectral weight in the limit of large transverse momentum [10-12].

For a scalar operator with Lifshitz scaling dimension $\nu$, a WKB computation demonstrated that for $\hat{\omega} \ll \nu^{-(z-1)}$ the exponential suppression of spectral weight is of the form [10]

$$
\chi=2 \operatorname{Im} G_{R} \sim \exp \left[-\frac{\sqrt{\pi} \Gamma(1 /(2 z-2))}{z \Gamma(z /(2 z-2))}\left(\frac{1}{\hat{\omega}}\right)^{1 /(z-1)}\right],
$$

where $\hat{\omega}=\omega /|\vec{k}|^{z}$. On the gravitational side of the duality, this suppression is a general feature of the Lifshitz geometry, while on the field theory side it is a direct consequence of Lifshitz scaling. In particular, exponential suppression in the limit $\hat{\omega} \rightarrow 0$ is simply a statement that excitations do not fall below the dispersion relation $\omega \sim|\vec{k}|^{z}$ [13]. (When $z=1$, the suppression is in fact complete, as $\chi=0$ for $\omega<|\vec{k}|$.)

While exponential suppression in the low-frequency limit is a robust feature of Lifshitz scaling, the actual suppression factor may a priori be operator and spin dependent. Thus we are motivated to investigate the spin- $1 / 2$ fermion Green's function in a pure Lifshitz geometry. We show that, although the square of the Dirac equation in the bulk does not coincide with the scalar Klein-Gordon equation, the suppression factor remains identical to that of the scalar in (1.3). Fermion correlators in AdS/CFT were investigated in [14-16], and further developed in [17-19]. Exponential suppression of fermion spectral weight has previously been noted in [10, 20-24], but is absent for vanishing bulk fermion mass [25, 26]. Exponential suppression in the current-current correlator was demonstrated in [11] and at non-zero temperature in the $T \rightarrow 0$ limit in [13, 27-29].

In section 2, we examine the Dirac equation and the holographic computation of the Green's function in a Lifshitz background. Then, in section 3, we compute the fermion spectral function using the WKB approximation. Taking the low frequency limit, $\hat{\omega} \rightarrow 0$, then reproduces the exponential suppression indicated in (1.3). Finally, we conclude in section 4 with an argument that the suppression factor in (1.3) is universal, regardless of spin and scaling dimension. 


\section{The Dirac equation in a Lifshitz background}

We consider a free Dirac particle in the bulk, with equation of motion

$$
\left(\Gamma^{M} \nabla_{M}-m\right) \psi=0
$$

The covariant derivative acting on $\psi$ is given by $\nabla_{M}=\partial_{M}+\frac{1}{4} \omega_{M}{ }^{\bar{N}} \bar{P} \Gamma_{\bar{N} \bar{P}}$, where $\omega_{M} \bar{N} \bar{P}$ is the spin connection, and bars indicate tangent space indices. To proceed, we note that the spin connection for a metric of the form

$$
d s^{2}=-e^{2 A(r)} d t^{2}+e^{2 B(r)} d \vec{x}_{n}^{2}+e^{2 C(r)} d r^{2},
$$

is especially simple. This allows us to rewrite the Dirac equation as

$$
\left(\Gamma^{M} \partial_{M}-m\right) \tilde{\psi}=0
$$

where $\tilde{\psi}=e^{\frac{1}{2}(A+n B)} \psi$. Note that, while the Dirac operator is written in terms of partial derivatives, it still corresponds to a curved background since the Dirac matrices are written in curved space. In particular

$$
\Gamma^{t}=e^{-A} \Gamma^{\bar{t}}, \quad \Gamma^{i}=e^{-B} \Gamma^{\bar{i}}, \quad \Gamma^{r}=e^{-C} \Gamma^{\bar{r}},
$$

where $i$ goes over the spatial coordinates.

Since the boundary theory is translationally invariant, we work in momentum space, and hence assume a plane wave solution of the form $\tilde{\psi}(t, \vec{x})=e^{i(\vec{k} \cdot \vec{x}-\omega t)} f(r)$. Inserting this into (2.3) and rearranging gives

$$
\left[\Gamma^{\bar{r}} \partial_{r}-m e^{C}-i\left(\omega e^{C-A} \Gamma^{\bar{t}}-k_{i} e^{C-B} \Gamma^{\bar{i}}\right)\right] f(r)=0 .
$$

At this point, it is worth recalling some basic facts about fermions in AdS/CFT. Specializing to the Lifshitz metric (1.2), where $e^{-A}=(r / L)^{z}$ and $e^{-B}=e^{-C}=r / L$, the Dirac equation becomes

$$
\left[\Gamma^{\bar{r}} \partial_{r}-\frac{m L}{r}-i\left(\omega\left(\frac{r}{L}\right)^{z-1} \Gamma^{\bar{t}}-k_{i} \Gamma^{\bar{i}}\right)\right] f(r)=0
$$

Taking $z>1$, the boundary (i.e. $r \rightarrow 0$ ) behavior is governed by $\left(\Gamma^{\bar{r}} \partial_{r}-m L / r\right) f(r) \approx 0$, which is solved by

$$
f^{(1)} \sim r^{-m L}, \quad f^{(2)} \sim r^{m L},
$$

where $f^{(1)}=-\Gamma^{\bar{r}} f^{(1)}$ and $f^{(2)}=\Gamma^{\bar{r}} f^{(2)}$ have definite $\Gamma^{\bar{r}}$ eigenvalues. Taking $m>0$ (which we assume throughout), this demonstrates that $f^{(1)}$ corresponds to the source, as it is non-normalizable as $r \rightarrow 0$, while $f^{(2)}$ corresponds to the response.

Unlike in the scalar case, the Dirac equation is first order, and can be thought of as relating half of the spinor components to the other half. From a holographic point of view, this implies that the CFT operator has half the spinor components as that of the bulk Dirac field [17]. For an even dimensional boundary theory, $f^{(1)}$ couples to a chiral fermionic operator (with $\Gamma^{\bar{r}}$ serving as the chirality matrix). For an odd dimensional 
boundary theory, the spinor components are halved simply because the Dirac representation is halved by going down in dimension.

Motivated by $(2.7)$, we now split $f(r)$ into source $(\eta)$ and response $(\xi)$ terms according to

$$
f=\eta-i \Gamma^{\bar{t}} \xi, \quad \Gamma^{\bar{r}} \eta=-\eta, \quad \Gamma^{\bar{r}} \xi=-\xi .
$$

The Dirac equation (2.6) then reduces to the system of coupled linear equations

$$
\begin{aligned}
& \left(\partial_{r}+m L / r\right) \eta=\left(\omega(r / L)^{z-1}-k_{i} \Gamma^{\overline{t \bar{i}}}\right) \xi, \\
& \left(\partial_{r}-m L / r\right) \xi=-\left(\omega(r / L)^{z-1}+k_{i} \Gamma^{\bar{t}}\right) \eta .
\end{aligned}
$$

These are matrix equations for the chiral spinors $\eta$ and $\xi$. To proceed, we note that $k_{i} \Gamma^{\overline{t i}}$ has eigenvalues $\pm k$. This allows us make the further projections

$$
\eta_{ \pm}=P_{ \pm} \eta, \quad \xi_{ \pm}=P_{ \pm} \xi
$$

where

$$
P_{ \pm}=\frac{1}{2}\left(1 \pm \frac{k_{i} \Gamma^{\overline{t i}}}{k}\right), \quad k \equiv|\vec{k}| .
$$

It is important to keep in mind that this is separate from (and commutes with) the $\Gamma^{\bar{r}}$ projection used above. The end result is a pair of coupled equations (one for $\eta_{+}$and $\xi_{+}$ and another for $\eta_{-}$and $\xi_{-}$)

$$
\begin{aligned}
& \left(\partial_{r}+m / r\right) \eta_{ \pm}=\left(\omega r^{z-1} \mp k\right) \xi_{ \pm}, \\
& \left(\partial_{r}-m / r\right) \xi_{ \pm}=-\left(\omega r^{z-1} \pm k\right) \eta_{ \pm},
\end{aligned}
$$

where we have set $L=1$. At this stage, we may note that component equations (2.12) are invariant under the interchange $\omega \rightarrow-\omega$ along with $\eta_{ \pm} \rightarrow \eta_{\mp}$ and $\xi_{ \pm} \rightarrow-\xi_{\mp}$. Since this relates positive and negative $\omega$, we may focus on $\omega>0$ and use this symmetry to handle the case of negative $\omega$.

The above equations can be recast in second-order form

$$
\begin{aligned}
& \left(\partial_{r}^{2}-\frac{\nu_{+}^{2}-1 / 4}{r^{2}}+\omega^{2} r^{2(z-1)}-k^{2}\right) \eta_{ \pm}-\frac{(z-1) \omega r^{z-2}}{\omega r^{z-1} \mp k}\left(\partial_{r}+\frac{m}{r}\right) \eta_{ \pm}=0 \\
& \left(\partial_{r}^{2}-\frac{\nu_{-}^{2}-1 / 4}{r^{2}}+\omega^{2} r^{2(z-1)}-k^{2}\right) \xi_{ \pm}-\frac{(z-1) \omega r^{z-2}}{\omega r^{z-1} \pm k}\left(\partial_{r}-\frac{m}{r}\right) \xi_{ \pm}=0
\end{aligned}
$$

where $\nu_{ \pm}=m \pm 1 / 2$. Finally, we transform to Schrödinger form by defining

$$
\eta_{ \pm}=\sqrt{\omega r^{z-1} \mp k} \Psi_{ \pm}^{(1)}, \quad \xi_{ \pm}=\sqrt{\omega r^{z-1} \pm k} \Psi_{ \pm}^{(2)} .
$$

We then obtain

$$
-\partial_{r}^{2} \Psi_{ \pm}^{(1)}+V_{ \pm}^{(1)} \Psi_{ \pm}^{(1)}=0, \quad-\partial_{r}^{2} \Psi_{ \pm}^{(2)}+V_{ \pm}^{(2)} \Psi_{ \pm}^{(2)}=0
$$


with effective potentials [24]

$$
\begin{aligned}
& V_{ \pm}^{(1)}=\frac{\nu_{+}^{2}-1 / 4}{r^{2}}-\omega^{2} r^{2(z-1)}+k^{2}+(z-1) \omega r^{z-3}\left(\frac{\nu_{+}}{\omega r^{z-1} \mp k}+\frac{z-1}{4} \frac{\omega r^{z-1} \pm 2 k}{\left(\omega r^{z-1} \mp k\right)^{2}}\right), \\
& V_{ \pm}^{(2)}=\frac{\nu_{-}^{2}-1 / 4}{r^{2}}-\omega^{2} r^{2(z-1)}+k^{2}+(z-1) \omega r^{z-3}\left(-\frac{\nu_{-}}{\omega r^{z-1} \pm k}+\frac{z-1}{4} \frac{\omega r^{z-1} \mp 2 k}{\left(\omega r^{z-1} \pm k\right)^{2}}\right) .
\end{aligned}
$$

Since these Schrödinger potentials were essentially obtained from the square of the Dirac equation, it is interesting to compare them to the corresponding potential for a bulk scalar. We see that the terms in (2.16) not proportional to $z-1$ are universal, and match those of the scalar potential

$$
V_{0}=\frac{\nu_{0}^{2}-1 / 4}{r^{2}}-\omega^{2} r^{2(z-1)}+k^{2}
$$

while the remaining terms are specific to the Dirac equation. Provided $z>1$, the boundary $(r \rightarrow 0)$ behavior is governed by the first terms in (2.16), which provides the proper scaling dimension of the dual operator. On the other side, the horizon behavior is dominated by $-\omega^{2} r^{2(z-1)}$. The additional terms in the potential will contribute to the bulk wavefunction, but will not substantially modify its qualitative features.

There is, however, a subtlety in the approach of using second order equations, and that is that the denominators in (2.16) or even (2.13) may blow up somewhere in the bulk. Since we focus on $\omega>0$, this happens in the $\Psi_{+}^{(1)}$ and $\Psi_{-}^{(2)}$ equations. (The magnitude of the wavevector $\vec{k}$ is always non-negative). Note also that the square-root factors in (2.14) can become imaginary on one side of the singularity.

Of course, we expect that the solution to the first order equations (2.12) ought to be well behaved at $\omega r^{z-1}-k=0$. Moreover, for $\omega>0$, the second order equations for $\Psi_{-}^{(1)}$ and $\Psi_{+}^{(2)}$ are well-behaved for $r>0$. This provides us with a well-defined procedure for solving the system. We first solve the effective Schrödinger problem for $\eta_{-}$and $\xi_{+}$, and then use the first order equations (2.12) to obtain $\eta_{+}$and $\xi_{-}$. This also has the benefit of guaranteeing that the first order equations are consistently solved without a doubling of the degrees of freedom that would arise from independent second order equations.

\subsection{Extracting the holographic Green's function}

We follow the procedure of [17] to obtain the fermionic Green's function from the solution to the bulk Dirac equation. The starting point is the boundary asymptotics of the Dirac field $\psi=r^{\frac{1}{2}(z+n)} f(r)$, which may be obtained by dropping the $\omega$ term in (2.6). As highlighted by (2.7), we split $\psi$ into components $\psi^{(1)}$ and $\psi^{(2)}$ with definite $\Gamma^{\bar{r}}$ eigenvalues. The Dirac equation can be converted to second-order form, with asymptotic behavior

$$
\begin{aligned}
& \psi^{(1)}=A r^{\frac{1}{2}(z+n)-m}+B r^{\frac{1}{2}(z+n)+m+1}, \\
& \psi^{(2)}=C r^{\frac{1}{2}(z+n)-m+1}+D r^{\frac{1}{2}(z+n)+m},
\end{aligned}
$$


as $r \rightarrow 0$. Here $A, B, C$ and $D$ are constant spinors that are related by the first order Dirac equation

$$
C=\frac{i k_{i} \Gamma^{\bar{i}}}{2 m-1} A, \quad B=\frac{i k_{i} \Gamma^{\bar{i}}}{2 m+1} D .
$$

The Green's function $G$ then relates the normalizable mode $(D)$ to the non-normalizable one $(A)$

$$
D=G\left(i \Gamma^{\bar{t}}\right) A .
$$

As usual, the retarded Green's function $G_{R}$ is obtained by taking infalling boundary conditions at the horizon.

Now suppose we have a solution to the effective Schrödinger problem (2.15) for $\Psi_{-}^{(1)}$ and $\Psi_{+}^{(2)}$ of the form

$$
\begin{aligned}
& \Psi_{-}^{(1)}=\alpha_{(1)} r^{-m}+\beta_{(1)} r^{m+1}, \\
& \Psi_{+}^{(2)}=\alpha_{(2)} r^{-m+1}+\beta_{(2)} r^{m},
\end{aligned}
$$

as $r \rightarrow 0$. We may then use (2.14) and (2.12) to translate this back to expressions for $\eta$ and $\xi$

$$
\begin{aligned}
& \eta_{+}=\frac{2 m-1}{k} \alpha_{(2)} \sqrt{k} r^{-m}-\frac{k}{2 m+1} \beta_{(2)} \sqrt{k} r^{m+1}, \\
& \eta_{-}=\alpha_{(1)} \sqrt{k} r^{-m}+\beta_{(1)} \sqrt{k} r^{m+1}, \\
& \xi_{+}=\alpha_{(2)} \sqrt{k} r^{-m+1}+\beta_{(2)} \sqrt{k} r^{m}, \\
& \xi_{-}=-\frac{k}{2 m-1} \alpha_{(1)} \sqrt{k} r^{-m+1}+\frac{2 m+1}{k} \beta_{(1)} \sqrt{k} r^{m} .
\end{aligned}
$$

Using the relation (2.8) then allows us to extract the coefficients

$$
\begin{aligned}
& D=-i \Gamma^{\bar{t}} \frac{1}{2}\left(1-\Gamma^{\bar{r}}\right) \sqrt{k}\left(P_{+} \beta_{(2)}+\frac{2 m+1}{k} P_{-} \beta_{(1)}\right), \\
& A=\frac{1}{2}\left(1-\Gamma^{\bar{r}}\right) \sqrt{k}\left(\frac{2 m-1}{k} P_{+} \alpha_{(2)}+P_{-} \alpha_{(1)}\right),
\end{aligned}
$$

where we have made the projections explicit. Comparing this with (2.20) then gives

$$
G_{R}=\frac{1}{2}\left(1+\Gamma^{\bar{r}}\right)\left(P_{+} G_{(1)}+P_{-} G_{(2)}\right)
$$

where

$$
G_{(1)}=-\frac{2 m+1}{k} \frac{\beta_{(1)}}{\alpha_{(1)}}, \quad G_{(2)}=-\frac{k}{2 m-1} \frac{\beta_{(2)}}{\alpha_{(2)}} .
$$

Note that, while $\alpha_{(i)}$ and $\beta_{(i)}$ are spinors, since there is a single effective Schrödinger equation governing each pair $\left\{\alpha_{(1)}, \beta_{(1)}\right\}$ and $\left\{\alpha_{(2)}, \eta_{(2)}\right\}$, the ratios $\beta_{(i)} / \alpha_{(i)}$ are well defined. Furthermore, the overall $\Gamma^{\bar{r}}$ projection is related to the fact that the boundary spinor degrees of freedom are half that of the bulk.

To summarize, the procedure for obtaining the holographic Green's function for $\omega>0$ is first to solve the effective Schrödinger equation (2.15) for $\Psi_{-}^{(1)}$ and $\Psi_{+}^{(2)}$, and then to 
extract the quantities $\alpha_{(i)}$ and $\beta_{(i)}$ from the boundary asymptotics according to (2.21). The Green's function then has the block diagonal form (2.24). For $\omega<0$, we use the symmetry properties of (2.12) to map

$$
G_{(1)}(-\omega, k)=-G_{(2)}^{*}(\omega, k), \quad G_{(2)}(-\omega, k)=-G_{(1)}^{*}(\omega, k)
$$

\section{The holographic Green's function in the WKB approximation}

We are now ready to examine the holographic Green's function. As we have seen above, we first solve the Dirac equation in the bulk, which can be transformed into the equivalent Schrödinger-like equations $(2.15)$ for $\Psi_{-}^{(1)}$ and $\Psi_{+}^{(2)}$. The effective potentials $V_{-}^{(1)}$ and $V_{+}^{(2)}$ given in (2.16) can be rewritten as

$$
\begin{aligned}
& V_{-}^{(1)}=\frac{\nu_{+}^{2}-1 / 4}{r^{2}}-\omega^{2} r^{2 \delta}+k^{2}+\frac{\delta}{r^{2}}\left(\omega r^{\delta}\right)\left(\frac{\nu_{+}}{\omega r^{\delta}+k}+\frac{\delta}{4} \frac{\omega r^{\delta}-2 k}{\left(\omega r^{\delta}+k\right)^{2}}\right), \\
& V_{+}^{(2)}=\frac{\nu_{-}^{2}-1 / 4}{r^{2}}-\omega^{2} r^{2 \delta}+k^{2}+\frac{\delta}{r^{2}}\left(\omega r^{\delta}\right)\left(-\frac{\nu_{-}}{\omega r^{\delta}+k}+\frac{\delta}{4} \frac{\omega r^{\delta}-2 k}{\left(\omega r^{\delta}+k\right)^{2}}\right),
\end{aligned}
$$

where $\delta \equiv z-1>0$. The first three terms in the potential are common to the scalar case, while the terms in the parentheses arise from squaring the Dirac equation. Note that the potential is not analytic in $r$ for non-integer $\delta$.

The behavior of the potentials near the boundary is given by

$$
\begin{aligned}
& V_{-}^{(1)}(r \rightarrow 0) \approx \frac{\nu_{+}^{2}-1 / 4}{r^{2}}+\frac{\omega \delta\left(\nu_{+}-\delta / 2\right) / k}{r^{2-\delta}}+\cdots . \\
& V_{+}^{(2)}(r \rightarrow 0) \approx \frac{\nu_{-}^{2}-1 / 4}{r^{2}}-\frac{\omega \delta\left(\nu_{-}+\delta / 2\right) / k}{r^{2-\delta}}+\cdots .
\end{aligned}
$$

These potentials remain dominated by the $1 / r^{2}$ term, which leads to the expected powerlaw boundary asymptotics $\Psi_{-}^{(1)} \sim r^{1 / 2 \pm \nu_{+}}$and $\Psi_{+}^{(2)} \sim r^{1 / 2 \pm \nu_{-}}$as indicated in (2.21). The horizon behavior, on the other hand, is universal, and arises from the divergent term

$$
V(r \rightarrow \infty) \sim-\omega^{2} r^{2 \delta}
$$

The horizon behavior is then of the form

$$
\Psi(r \rightarrow \infty) \sim r^{-\delta / 2} e^{ \pm i \omega r^{z} / z}
$$

The retarded Green's function is obtained by taking the positive sign, corresponding to infalling boundary conditions.

In general, it is not possible to obtain a closed-form solution to the Schrödinger equation with potential given by (3.1). However, we can search for tunneling barriers, and hence regions of exponential suppression, using the WKB approximation. Following ref. [12], 
which analyzed the scalar case, we assume the potential $V$ admits a single turning point $r_{0}$ with $V\left(r_{0}\right)=0$. The first order WKB wavefunction is then given by

$\Psi_{\mathrm{WKB}}(r)= \begin{cases}\frac{1}{\hat{V}(r)^{\frac{1}{4}}}\left[F \exp \left(\int_{r}^{r_{0}} d r^{\prime} \sqrt{\hat{V}\left(r^{\prime}\right)}\right)+G \exp \left(-\int_{r}^{r_{0}} d r^{\prime} \sqrt{\hat{V}\left(r^{\prime}\right)}\right)\right], & r<r_{0} ; \\ \frac{1}{|\hat{V}(r)|^{\frac{1}{4}}}\left[a \exp \left(i \int_{r_{0}}^{r} d r^{\prime} \sqrt{\left|\hat{V}\left(r^{\prime}\right)\right|}\right)+b \exp \left(-i \int_{r_{0}}^{r} d r^{\prime} \sqrt{\left|\hat{V}\left(r^{\prime}\right)\right|}\right)\right], \quad r>r_{0},\end{cases}$

where $\hat{V}(r)=V(r)+1 / 4 r^{2}$. This shift arises when applying the WKB method to a potential that blows up as $1 / r^{2}$, and corresponds to making the shift $\nu_{ \pm}^{2}-1 / 4 \rightarrow \nu_{ \pm}^{2}$ in the first term of the potential in (3.1). See appendix A for details.

The boundary behavior, encoded by the constants $F$ and $G$, is related to the horizon behavior, encoded by the constants $a$ and $b$, via the WKB connection formulae

$$
\left(\begin{array}{c}
F \\
G
\end{array}\right)=\left(\begin{array}{cc}
e^{-i \pi / 4} & e^{i \pi / 4} \\
\frac{1}{2} e^{i \pi / 4} & \frac{1}{2} e^{-i \pi / 4}
\end{array}\right)\left(\begin{array}{l}
a \\
b
\end{array}\right) .
$$

In order to compute the holographic Green's function (2.24), we need to relate the boundary coefficients $\alpha_{(i)}$ and $\beta_{(i)}$ defined in (2.21) to the WKB coefficients $F$ and $G$. Using the boundary behavior $\hat{V} \approx \nu^{2} / r^{2}$, we see that

$$
\Psi_{\mathrm{WKB}}(r \rightarrow 0) \sim F c_{1} r^{1 / 2-\nu}+G c_{2} r^{1 / 2+\nu},
$$

where $c_{1}$ and $c_{2}$ are constants obtained by integrating up to the classical turning point. This demonstrates that $F$ is related to the non-normalizable mode with coefficient $\alpha_{(i)}$ and $G$ is related to the normalizable mode with coefficient $\beta_{(i)}$. This allows us to obtain

$$
\begin{aligned}
& \alpha_{(i)}=\lim _{r \rightarrow 0} \frac{F r^{\nu-1 / 2}}{\hat{V}(r)^{\frac{1}{4}}} \exp \left(\int_{r}^{r_{0}} d r^{\prime} \sqrt{\hat{V}\left(r^{\prime}\right)}\right), \\
& \beta_{(i)}=\lim _{r \rightarrow 0} \frac{G r^{-\nu-1 / 2}}{\hat{V}(r)^{\frac{1}{4}}} \exp \left(-\int_{r}^{r_{0}} d r^{\prime} \sqrt{\hat{V}\left(r^{\prime}\right)}\right) .
\end{aligned}
$$

At this point, it is worth noting that while the term proportional to $F$ in the WKB approximation is dominated by the non-normalizable mode, in fact it may contain a subdominant (and in general non-analytic) piece related to the normalizable mode. As discussed in [12], the WKB approximation is unreliable in extracting this subdominant behavior. However, this only affects the real part of the holographic Green's function, and in particular the imaginary part remains under control. In any case, what this means is that only the component of $\beta_{(i)}$ in (3.8) that is orthogonal to $\alpha_{(i)}$ in the complex plane is to be trusted.

For the retarded Green's function, we take infalling boundary conditions, which corresponds to setting $b=0$ in (3.6). The holographic Green's function is then given by (2.24) where

$$
\frac{\beta_{(i)}}{\alpha_{(i)}}=\frac{i}{2} \lim _{r \rightarrow 0} r^{-2 \nu} \exp \left(-2 \int_{r}^{r_{0}} d r^{\prime} \sqrt{\hat{V}\left(r^{\prime}\right)}\right) .
$$

Here $\nu$ is taken to be $\nu_{+}$and $\nu_{-}$, while $\hat{V}(r)$ is $\hat{V}_{-}^{(1)}$ and $\hat{V}_{+}^{(2)}$, respectively, for $i=1$ and $i=2$. Note that this quantity is manifestly imaginary, and contributes to the spectral 
function. The real part of the Green's function is actually non-vanishing, but cannot be obtained by this WKB method.

Since the WKB approximation is reliable for the imaginary part of the Green's function, we focus on the spectral function, defined as

$$
\chi=-\frac{1}{\pi} \operatorname{Im} \operatorname{Tr} G_{R}=-\frac{2^{\lfloor n / 2\rfloor}}{2 \pi} \operatorname{Im}\left(G_{(1)}+G_{(2)}\right),
$$

where $G_{(1)}$ and $G_{(2)}$ are given in (2.25), and may be obtained from (3.9). (The normalization factor is related to the bulk dimension $n+2$.) For $k \neq 0$, we may transform the expression (3.9) into a dimensionless WKB integral by letting $x=k r$. The result is then

$$
\begin{aligned}
& \operatorname{Im} G_{(1)}=-\frac{2 m+1}{2} k^{2 m} \lim _{\epsilon \rightarrow 0} \epsilon^{-2 \nu_{+}} \exp \left(-2 \int_{\epsilon}^{x_{0}} d x^{\prime} \sqrt{\hat{V}_{-}^{(1)}\left(x^{\prime}\right)}\right), \\
& \operatorname{Im} G_{(2)}=-\frac{1}{2(2 m-1)} k^{2 m} \lim _{\epsilon \rightarrow 0} \epsilon^{-2 \nu_{-}} \exp \left(-2 \int_{\epsilon}^{x_{0}} d x^{\prime} \sqrt{\hat{V}_{+}^{(2)}\left(x^{\prime}\right)}\right),
\end{aligned}
$$

where

$$
\begin{aligned}
& \hat{V}_{-}^{(1)}(x)=\frac{\nu_{+}^{2}}{x^{2}}-\hat{\omega}^{2} x^{2 \delta}+1+\frac{\delta}{x^{2}} \hat{\omega} x^{\delta}\left(\frac{\nu_{+}}{\hat{\omega} x^{\delta}+1}+\frac{\delta}{4} \frac{\hat{\omega} x^{\delta}-2}{\left(\hat{\omega} x^{\delta}+1\right)^{2}}\right), \\
& \hat{V}_{+}^{(2)}(x)=\frac{\nu_{-}^{2}}{x^{2}}-\hat{\omega}^{2} x^{2 \delta}+1+\frac{\delta}{x^{2}} \hat{\omega} x^{\delta}\left(-\frac{\nu_{-}}{\hat{\omega} x^{\delta}+1}+\frac{\delta}{4} \frac{\hat{\omega} x^{\delta}-2}{\left(\hat{\omega} x^{\delta}+1\right)^{2}}\right) .
\end{aligned}
$$

Here we have defined the scale-invariant quantity $\hat{\omega}=\omega / k^{z}$.

The expressions (3.11) are valid for $\hat{\omega}>0$. The behavior for $\hat{\omega}<0$, may be obtained using (2.26). While in the above we have focused on first order WKB, the analysis can be extended to higher order as well. The second order correction is presented in appendix A, and as an example we show a comparison of the first and second order approximations with the exact numerical solution in figure 1. Details of how we obtained the numerical solution are given in appendix B. Although there is considerable deviation of the first order WKB result from the exact solution for negative $\hat{\omega}$, it agrees well in the exponential suppression region around $\hat{\omega} \approx 0$.

\subsection{Exponential suppression in the $\hat{\omega} \rightarrow 0$ limit}

Although the WKB potential (3.12) is somewhat complicated, as already seen in (2.17), the first three terms match that of the bulk scalar. In fact, in the limit $\hat{\omega} \rightarrow 0$, only these first three terms are important, and the spin- $1 / 2$ potential reduces to that of the spin- 0 case. As a result, it immediately follows that the exponential suppression of spectral weight in the zero frequency limit is identical for spin- 0 and spin- $1 / 2$ operators.

In particular, a matched asymptotic expansion gives the result [10]

$$
\operatorname{Im} G_{(1,2)} \approx-\frac{e^{2 \nu_{ \pm}}}{2}\left(\frac{k}{2 \nu_{ \pm}}\right)^{2 m} \exp \left(-\frac{\sqrt{\pi} \Gamma(1 / 2 \delta)}{z \hat{\omega}^{1 / \delta} \Gamma(z / 2 \delta)}\right)
$$

in the limit $\hat{\omega} \ll \nu^{-\delta}$, where we recall that $\delta=z-1$. The suppression factor agrees with that of the spin-0 case, (1.3), as it must, since the WKB potential is identical in the zero frequency limit. The asymptotic behavior in this limit also agrees with the Green's function extracted from the numerical solution of the Dirac equation, (2.12) (see figure 1). 


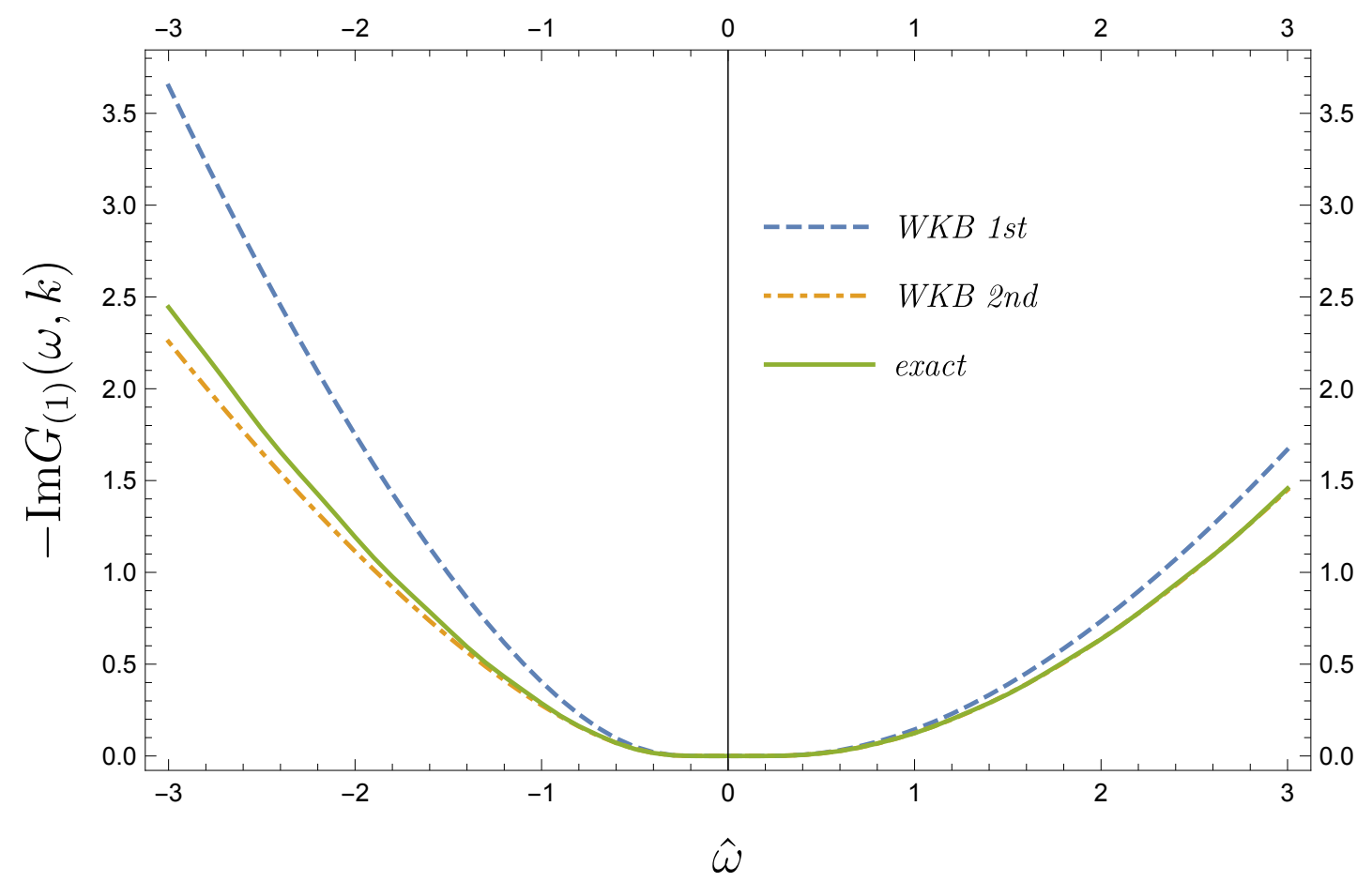

Figure 1. Plot of $-\operatorname{Im} G_{(1)}(\omega, k)$ with $z=2, m=1.55$, and $k=1$. The three lines represent calculations from first-order WKB, second-order WKB, and the exact numerical solution. The flat region near $\hat{\omega} \approx 0$ features the exponential suppression. One may obtain a plot of $-\operatorname{Im} G_{(2)}(\omega, k)$ by flipping $\hat{\omega} \rightarrow-\hat{\omega}$.

\section{Discussion}

While it may be natural to generalize relativistic holography to the non-relativistic case by, for example, introducing a Lifshitz bulk of the form (1.2), the lack of boost invariance can have a profound effect on the dynamics of AdS/CFT. At a classical level, the fact that non-radial null geodesics no longer reach the boundary suggests a decoupling of the bulk and boundary, leading to 'trapped' modes that cannot be probed by local observables in the boundary field theory. This classical intuition was confirmed in $[9,12]$ for the case of bulk scalars, and, as we have shown above, generalizes to bulk fermions as well.

In both scalar and fermion cases, at leading order, the bulk fields satisfy an effective Schrödinger equation with a potential of the form

$$
\hat{V}(x)=\frac{\nu^{2}}{x^{2}}-\hat{\omega}^{2} x^{2(z-1)}+1+\cdots .
$$

As we approach the boundary $(x \rightarrow 0)$, the first term in the potential dominates, and the wavefunction is a power-law, as appropriate for scale-invariant behavior. On the other hand, the horizon $(x \rightarrow \infty)$ behavior is governed by the second term. Provided $z>1$ (i.e. the non-relativistic case), the potential drops to $-\infty$, and the wavefunction is oscillatory. For $\hat{\omega} \ll \nu^{-(z-1)}$, an intermediate region develops with $\hat{V} \approx 1$. Since the effective potential is referenced to zero energy, this gives rise to a tunneling barrier, and elementary considera- 
tions demonstrates that the tunneling amplitude is of the form $e^{-s}$ where $s \approx-2 / \hat{\omega}^{1 /(z-1)}$. A more careful WKB analysis the gives rise to the expression (1.3).

Based on the experience with the effective Schrödinger potential for spin-0 and spin$1 / 2$ fields in the bulk, we expect that the result is universal and independent of spin. This can be verified for the case of arbitrary integer spin. Consider, for example, a spin- $\ell$ field described by a completely symmetric and trace-free field $\Phi_{\left(M_{1} M_{2} \cdots M_{\ell}\right)}$ satisfying

$$
\left(\square-m^{2}\right) \Phi_{M_{1} M_{2} \cdots M_{\ell}}=0, \quad \nabla^{N} \Phi_{N M_{2} \cdots M_{\ell}}=0, \quad \Phi^{N}{ }_{N M_{3} \cdots M_{\ell}}=0 .
$$

Specializing to the Lifshitz metric (1.2) and taking only the space components $\Phi_{i_{1} \cdots i_{\ell}}$ nonvanishing gives the equation of motion

$$
\left[\partial_{r}^{2}-\frac{n+z-2 \ell-1}{r} \partial_{r}-\frac{m^{2}+\ell(n+z-\ell+1)}{r^{2}}+\omega^{2} r^{2(z-1)}-k^{2}\right] \Phi_{i_{1} \cdots i_{\ell}}=0,
$$

along with the transverse condition $k^{j} \Phi_{j i_{2} \cdots i_{\ell}}=0$. This can be converted to Schrödinger form by defining

$$
\Psi_{i_{1} \cdots i_{\ell}}=r^{-(n+z-2 \ell-1) / 2} \Phi_{i_{1} \cdots i_{\ell}} .
$$

The effective Schrödinger equation is then $\left(-\partial_{r}^{2}+V_{\ell}\right) \Psi_{i_{1} \cdots i_{\ell}}=0$ where

$$
V_{\ell}=\frac{\nu_{\ell}^{2}-1 / 4}{r^{2}}-\omega^{2} r^{2(z-1)}+k^{2}, \quad \nu_{\ell}^{2}=\ell+\left(\frac{n+z}{2}\right)^{2}+m^{2} .
$$

This reduces to the scalar case (2.17) when $\ell=0$.

Although the scaling dimension $\nu_{\ell}$ (or equivalently bulk mass) in (4.5) governs the power-law behavior of the Green's function, the exponential suppression arises from the interplay between the $\omega^{2}$ and $k^{2}$ terms, and hence is independent of $\nu_{\ell}$. In particular, the low-frequency suppression of spectral weight depends only on the critical exponent $z$ and the scale-invariant quantity $\hat{\omega}$. This matches the expectation that the exponential suppression is universal feature of Lifshitz scaling and is independent of the detailed dynamics of the field theory operators or their bulk dual fields.

Of course, the effective potential (3.12), which arises from squaring the Dirac equation, includes additional terms that are not present for integer spin fields. Note, however, that they drop out in the relativistic $(z=1)$ case, so the potential becomes independent of spin. This is easily understood from relativistic conformal invariance, which uniquely determines the Green's function, at least up to kinematical factors related to spin. Moving away from $z=1$, on the other hand, relaxes the form of the Green's function, and in particular allows it to have the behavior $G \sim k^{2 \nu z} f(\hat{\omega})$, where $f(\hat{\omega})$ is a function of the scale-invariant quantity $\hat{\omega}$. Although the solution to the Dirac equation in a pure Lifshitz background fully determines this function, it will receive modifications once we introduce higher derivative corrections in the bulk [30].

Just as in the scalar case, such correction terms will relax the form of the Green's function. Nevertheless, the exponential suppression of spectral weight at low frequencies is universal, and remains robust, even in the presence of higher derivative corrections, so 
long as $\hat{\omega} \gg\left(\ell_{*} / L\right)^{z-1}$. Here $L$ is the Lifshitz curvature scale in (1.2) and $\ell_{*}$ sets the scale of the higher derivative expansion.

Finally, although we have focused on the scale-invariant case, our analysis carries over to more general bulk geometries with broken boost invariance. The main features of the holographic Green's function can be seen by the shape of the effective Schrödinger potential (3.1), and in particular the presence or absence of any tunneling barriers. Unlike for the scalar case, where the dependence of the effective potential on $\omega$ and $k$ is straightforward, the Dirac case has a rather complicated behavior in these parameters. For Lifshitz backgrounds, the additional terms in (3.1) are never dominant. However, this is not always the case for more general backgrounds. As a result, the scalar and fermion Green's functions can exhibit rather different behavior; this allows for such features as holographic fermi surfaces that are not present in the bosonic case. It would be interesting to explore and classify the structure of potential barriers in the more general setting and to relate the resulting suppression of spectral weight to the physics of the dual field theory.

\section{Acknowledgments}

JTL wishes to thank C. Keeler, G. Knodel and K. Sun for useful discussions. This work was supported in part by the US Department of Energy under grant DE-SC0007859.

\section{A The WKB approximation for $1 / r^{2}$ potentials}

In this appendix, we review the second order WKB approximation and furthermore apply it to the case of a $1 / r^{2}$ potential. Our starting point is the second order equation

$$
-\epsilon^{2} y^{\prime \prime}(x)+Q(x) y(x)=0,
$$

where we consider $\epsilon$ to be our expansion parameter. We then expand

$$
y(x) \sim \exp \left[\frac{1}{\epsilon} \sum_{n \geq 0} \epsilon^{n} S_{n}(x)\right] .
$$

Substituting the series expansion into (A.1) and matching powers of $\epsilon$ gives

$$
\begin{aligned}
& S_{0}^{\prime}= \pm \sqrt{Q}, \\
& S_{1}^{\prime}=-\frac{S_{0}^{\prime \prime}}{2 S_{0}^{\prime}}=-\frac{1}{2} \frac{d}{d x} \log \left(S_{0}^{\prime}\right), \\
& S_{2}^{\prime}=-\frac{S_{1}^{\prime \prime}+S_{1}^{\prime 2}}{2 S_{0}^{\prime}}
\end{aligned}
$$

and so on. In general, the terms $S_{n}$ are total derivatives for odd $n$, but not for even $n$. Integrating $S_{0}^{\prime}$ and $S_{2}^{\prime}$ allows us to write the second order WKB wavefunction

$$
y(x) \sim Q^{-1 / 4} \exp \left[ \pm \frac{1}{\epsilon} \int^{x} \sqrt{Q} d x \pm \epsilon\left(\frac{5 Q^{\prime}}{48 Q^{3 / 2}}+\int^{x} \frac{Q^{\prime \prime}}{48 Q^{3 / 2}} d x\right)+\mathcal{O}\left(\epsilon^{2}\right)\right]
$$

where we have integrated $S_{2}^{\prime}$ by parts. Ignoring the $\mathcal{O}(\epsilon)$ term in the exponent gives the usual first order WKB approximation. 


\section{A.1 Connection formulae}

The above expression is general for both classically forbidden $(Q>0)$ and classically allowed $(Q<0)$ regions, provided we allow the factors to become complex. (Up to second order, the exponent is a pure phase in the classically allowed region, although this does persist at higher orders.) Since the approximation breaks down at a turning point, we use a matched asymptotic expansion in order to connect solutions across the turning point. For the effective Schrödinger potentials we are interested in, we take $Q$ positive for $x<0$ and $Q$ negative for $x>0$. Here we have chosen the turning point to be $x=0$ for simplicity.

To fix the constants, we take

$$
y(x)=Q^{-1 / 4}\left\{\begin{array}{l}
F \\
G
\end{array}\right\} \exp \left[ \pm \frac{1}{\epsilon} \int_{x}^{0} \sqrt{Q} d x \mp \epsilon\left(\frac{5 Q^{\prime}}{48 Q^{3 / 2}}-\int_{x}^{-\mu} \frac{Q^{\prime \prime}}{48 Q^{3 / 2}} d x+\frac{\beta}{12(-\alpha)^{3 / 2} \sqrt{\mu}}\right)\right],
$$

on the left (i.e. for $x<0$ ). The last factor requires some explanation. Since the integrand $Q^{\prime \prime} / Q^{3 / 2}$ is singular at the turning point, we cannot integrate all the way to $x=0$. Instead, we regulate the integral by a small cutoff $\mu \rightarrow 0^{+}$. The last term then cancels the divergence where $a$ and $b$ are Taylor coefficients near the turning point

$$
Q(x)=\alpha x+\beta x^{2}+\cdots \quad \text { for } x \rightarrow 0 .
$$

Note that $\alpha<0$ since $Q$ has a negative slope going through the turning point.

For the solution on the right $(x>0)$, we take

$$
y(x)=|Q|^{-1 / 4}\left\{\begin{array}{l}
a \\
b
\end{array}\right\} \exp \left[ \pm \frac{i}{\epsilon} \int_{0}^{x} \sqrt{|Q|} d x \mp i \epsilon\left(\frac{5|Q|^{\prime}}{48|Q|^{3 / 2}}+\int_{\mu}^{x} \frac{|Q|^{\prime \prime}}{48|Q|^{3 / 2}} d x+\frac{\beta}{12(-\alpha)^{3 / 2} \sqrt{\mu}}\right)\right] \text {. }
$$

We match the left and right expressions by solving the equation

$$
-\epsilon^{2} y^{\prime \prime}+\left(\alpha x+\beta x^{2}+\cdots\right) y=0
$$

near the turning point. Ignoring $\beta$ gives an Airy function, while including $\beta$ and working to the next order gives

$$
y=\left(1-\frac{\beta x}{5 \alpha}+\cdots\right)\left\{\begin{array}{l}
\mathrm{Ai} \\
\mathrm{Bi}
\end{array}\right\}\left(-\epsilon^{-2 / 3}(-\alpha)^{1 / 3} x\left(1+\frac{\beta x}{5 \alpha}+\cdots\right)\right) .
$$

This expression is for $\alpha<0$. (For $\alpha>0$, the initial part of the argument of the Airy function should be replaced by $+\epsilon^{-2 / 3} \alpha^{1 / 3} x$.)

The connection formula between the forbidden and allowed solutions may be obtained by asymptotic expansion of (A.5), (A.7) and (A.9). It turns out that the expression is identical to that of first order WKB

$$
\left(\begin{array}{l}
F \\
G
\end{array}\right)=\left(\begin{array}{cc}
e^{-i \pi / 4} & e^{i \pi / 4} \\
\frac{1}{2} e^{i \pi / 4} & \frac{1}{2} e^{-i \pi / 4}
\end{array}\right)\left(\begin{array}{l}
a \\
b
\end{array}\right)
$$




\section{A.2 Handling the singular $1 / r^{2}$ potential}

In order for WKB to be a controlled expansion, we want the terms $S_{n}$ to be well behaved as an asymptotic series. This fails when we have $Q(x) \sim 1 / x^{2}$. Consider, for example $Q=(\tilde{\nu} / x)^{2}$. Then the expansion (A.3) gives

$$
S_{0}=\tilde{\nu} \log x, \quad S_{1}=\frac{1}{2} \log x, \quad S_{2}=\frac{1}{8 \tilde{\nu}} \log x,
$$

where we have taken the positive root. In this case, the higher order terms are just as important as the first order terms, and cannot systematically be dropped.

In order to handle a $1 / r^{2}$ potential, consider the Schrödinger equation

$$
-\frac{d^{2} \psi(r)}{d r^{2}}+V(r) \psi(r)=0 .
$$

We now make the transformation

$$
r=e^{x}, \quad \psi(r)=e^{x / 2} y(x) .
$$

This transforms the Schrödinger equation into

$$
-y^{\prime \prime}(x)+Q(x) y(x)=0
$$

where

$$
Q(x)=r^{2} V(r)+\left.\frac{1}{4}\right|_{r=e^{x}}
$$

We have thus transformed the singular potential into an asymptotically constant one

$$
V(r) \sim \frac{\nu^{2}-\frac{1}{4}}{r^{2}} \text { as } r \rightarrow 0 \quad \Rightarrow \quad Q(x) \sim \nu^{2} \text { as } x \rightarrow-\infty .
$$

This allows us to use the WKB approximation for the $Q(x)$ potential.

Transforming $y(x)$ in (A.5) back to $\psi(r)$ gives

$$
\begin{aligned}
\psi(r)= & \hat{V}^{-1 / 4}\left\{\begin{array}{l}
F \\
G
\end{array}\right\} \exp \left[ \pm \frac{1}{\epsilon} \int_{r}^{r_{0}} \sqrt{\hat{V}} d r\right. \\
& \left.\mp \epsilon\left(\frac{5\left(\hat{V}^{\prime}+\frac{2}{r} \hat{V}\right)}{48 \hat{V}^{3 / 2}}-\int_{r}^{r_{0}-\mu} \frac{\hat{V}^{\prime \prime}+\frac{5}{r} \hat{V}^{\prime}+\frac{4}{r^{2}} \hat{V}}{48 \hat{V}^{3 / 2}} d r+\frac{\frac{5 \alpha}{2 r_{0}}+\beta}{12(-\alpha)^{3 / 2} \mu^{1 / 2}}\right)\right],
\end{aligned}
$$

where

$$
\hat{V}=V+\frac{1}{4 r^{2}} .
$$

Here $r_{0}$ is the classical turning point and

$$
\hat{V}=\alpha\left(r-r_{0}\right)+\beta\left(r-r_{0}\right)^{2}+\cdots \quad \text { as } \quad r \rightarrow r_{0} .
$$

In particular, $\alpha=\hat{V}^{\prime}\left(r_{0}\right)$ and $\beta=\hat{V}^{\prime \prime}\left(r_{0}\right) / 2$.

One thing to note is that the terms in the small $\epsilon$ expansion are rearranged between the $\psi(r)$ equation and the $y(x)$ equation. If we put in an explicit $\epsilon^{2}$ in the second derivative term of the Schrödinger equation (A.12), then the $1 / 4$ term in the potential $Q(x)$ of (A.15) would become $\epsilon^{2} / 4$. However, since we are interested in $\epsilon=1$, the $\epsilon$ independent shift by $1 / 4$ is appropriate. 


\section{B The Green's function from the numerical method}

In this appendix, we explain the procedure to compute the imaginary part of the Green's function from the numerical solution of the Dirac equation. We follow the approach of $[18,31]$ and convert the coupled linear equations resulting from the components of the Dirac equation into a first order Riccati flow equation.

We defined $\eta$ and $\xi$ in (2.8) and showed that their projections, $\eta_{ \pm}=P_{ \pm} \eta$ and $\xi_{ \pm}=P_{ \pm} \xi$, follow the pair of coupled equations (2.12), which we repeat here:

$$
\begin{aligned}
& \left(\partial_{r}+m / r\right) \eta_{ \pm}=\left(\omega r^{z-1} \mp k\right) \xi_{ \pm}, \\
& \left(\partial_{r}-m / r\right) \xi_{ \pm}=-\left(\omega r^{z-1} \pm k\right) \eta_{ \pm} .
\end{aligned}
$$

The boundary behavior of $\eta_{ \pm}$and $\xi_{ \pm}$is

$$
\begin{aligned}
& \eta_{ \pm}=\alpha_{ \pm} r^{-m}+\beta_{ \pm} r^{m+1} \\
& \xi_{ \pm}=\gamma_{ \pm} r^{-m+1}+\delta_{ \pm} r^{m}
\end{aligned}
$$

as $r \rightarrow 0$, while the horizon behavior is

$$
\eta_{ \pm}=a e^{ \pm i \omega r^{z} / z}, \quad \xi_{ \pm}= \pm i a e^{ \pm i \omega r^{z} / z},
$$

as $r \rightarrow \infty$.

Although $\eta_{ \pm}$and $\xi_{ \pm}$are spinors, their equations of motion (B.1) do not contain matrices, i.e., do not mix the spinor components. Therefore, we may consider each of these spinors as a single function. This allows us to define the ratio $\zeta_{ \pm}=\xi_{ \pm} / \eta_{ \pm}$. The differential equation governing this ratio $\zeta_{ \pm}$is given by

$$
\partial_{r} \zeta_{ \pm}+\left(\omega r^{z-1} \mp k\right) \zeta_{ \pm}^{2}-\frac{2 m}{r} \zeta_{ \pm}+\left(\omega r^{z-1} \pm k\right)=0 .
$$

This ratio $\zeta_{ \pm}$exhibits boundary asymptotic behavior

$$
\zeta_{ \pm}=\frac{\gamma_{ \pm}}{\alpha_{ \pm}} r+\frac{\delta_{ \pm}}{\alpha_{ \pm}} r^{2 m}
$$

as $r \rightarrow 0$, and horizon asymptotic behavior

$$
\zeta_{ \pm}=i, \quad \text { or } \quad \zeta_{ \pm}=-i
$$

as $r \rightarrow \infty$. We will take $\zeta_{ \pm}$to be $i$ at the horizon, which corresponds to the infalling boundary condition. We note that each pair $\left\{\alpha_{ \pm}, \gamma_{ \pm}\right\}$and $\left\{\beta_{ \pm}, \delta_{ \pm}\right\}$in (B.5) is not independent. They are related by the coupled equations (B.1) as

$$
\gamma_{ \pm}=\frac{\mp k}{-2 m+1} \alpha_{ \pm}, \quad \beta_{ \pm}=\frac{\mp k}{2 m+1} \delta_{ \pm}
$$

in the limit $r \rightarrow 0$. The first relation shows that the ratio $\gamma_{ \pm} / \alpha_{ \pm}$is purely real. This means that we can pull out the imaginary part of the $\delta_{ \pm} / \alpha_{ \pm}$term by

$$
\operatorname{Im} \frac{\delta_{ \pm}}{\alpha_{ \pm}}=\lim _{r \rightarrow 0} \operatorname{Im} \zeta_{ \pm} r^{-2 m}
$$


This quantity can be used to obtain the imaginary part of the Green's function as done in section 2.1. Using the definition (2.8) to obtain $f_{ \pm}$and comparing to the asymptotic equations (2.18), we find

$$
A=\frac{1}{2}\left(1-\Gamma^{\bar{r}}\right)\left(P_{+} \alpha_{-}+P_{-} \alpha_{+}\right), \quad D=-i \Gamma^{\bar{t}} \frac{1}{2}\left(1-\Gamma^{\bar{r}}\right)\left(P_{+} \delta_{-}+P_{-} \delta_{+}\right)
$$

where we show the projection explicitly. The Green's function relates these quantities as specified by the equation (2.20), from which we obtain the imaginary part of the Green's function

$$
\operatorname{Im} G_{R}=-\operatorname{Im} \frac{1}{2}\left(1+\Gamma^{\bar{r}}\right)\left(P_{+} \frac{\delta_{-}}{\alpha_{-}}+P_{-} \frac{\delta_{+}}{\alpha_{+}}\right) .
$$

In brief, we have the following procedure to obtain the imaginary part of the Green's function. We seek the numerical solution of the differential equation for $\zeta_{ \pm}$, (B.4), with the initial condition, $\zeta_{ \pm}(r \rightarrow \infty)=i$. Then we evaluate $\zeta_{ \pm}(r \rightarrow 0)$ to obtain the ratio $\operatorname{Im} \delta_{ \pm} / \alpha_{ \pm}$from (B.8). Finally, the expression (B.10) gives us $\operatorname{Im} G_{R}$.

Open Access. This article is distributed under the terms of the Creative Commons Attribution License (CC-BY 4.0), which permits any use, distribution and reproduction in any medium, provided the original author(s) and source are credited.

\section{References}

[1] S. Kachru, X. Liu and M. Mulligan, Gravity duals of Lifshitz-like fixed points, Phys. Rev. D 78 (2008) 106005 [arXiv:0808.1725] [InSPIRE].

[2] S.A. Hartnoll, Lectures on holographic methods for condensed matter physics, Class. Quant. Grav. 26 (2009) 224002 [arXiv: 0903.3246] [INSPIRE].

[3] K. Copsey and R. Mann, Pathologies in asymptotically Lifshitz spacetimes, JHEP 03 (2011) 039 [arXiv: 1011.3502] [INSPIRE].

[4] G.T. Horowitz and B. Way, Lifshitz singularities, Phys. Rev. D 85 (2012) 046008 [arXiv:1111.1243] [INSPIRE].

[5] S. Harrison, S. Kachru and H. Wang, Resolving Lifshitz horizons, JHEP 02 (2014) 085 [arXiv: 1202.6635] [INSPIRE].

[6] N. Bao, X. Dong, S. Harrison and E. Silverstein, The Benefits of Stress: Resolution of the Lifshitz Singularity, Phys. Rev. D 86 (2012) 106008 [arXiv:1207.0171] [INSPIRE].

[7] J. Bhattacharya, S. Cremonini and A. Sinkovics, On the IR completion of geometries with hyperscaling violation, JHEP 02 (2013) 147 [arXiv:1208.1752] [INSPIRE].

[8] G. Knodel and J.T. Liu, Higher derivative corrections to Lifshitz backgrounds, JHEP 10 (2013) 002 [arXiv:1305.3279] [INSPIRE].

[9] C. Keeler, G. Knodel and J.T. Liu, What do non-relativistic CFTs tell us about Lifshitz spacetimes?, JHEP 01 (2014) 062 [arXiv:1308.5689] [INSPIRE].

[10] T. Faulkner and J. Polchinski, Semi-holographic Fermi liquids, JHEP 06 (2011) 012 [arXiv: 1001.5049] [INSPIRE].

[11] S.A. Hartnoll and E. Shaghoulian, Spectral weight in holographic scaling geometries, JHEP 07 (2012) 078 [arXiv: 1203.4236] [INSPIRE]. 
[12] C. Keeler, G. Knodel and J.T. Liu, Hidden horizons in non-relativistic AdS/CFT, JHEP 08 (2014) 024 [arXiv: 1404.4877] [INSPIRE].

[13] S.A. Hartnoll and D.M. Hofman, Locally Critical Resistivities from Umklapp Scattering, Phys. Rev. Lett. 108 (2012) 241601 [arXiv:1201.3917] [INSPIRE].

[14] M. Henningson and K. Sfetsos, Spinors and the AdS/CFT correspondence, Phys. Lett. B 431 (1998) 63 [hep-th/9803251] [INSPIRE].

[15] W. Mueck and K.S. Viswanathan, Conformal field theory correlators from classical field theory on anti-de Sitter space. 2. Vector and spinor fields, Phys. Rev. D 58 (1998) 106006 [hep-th/9805145] [INSPIRE].

[16] M. Henneaux, Boundary terms in the AdS/CFT correspondence for spinor fields, hep-th/9902137 [INSPIRE].

[17] N. Iqbal and H. Liu, Real-time response in AdS/CFT with application to spinors, Fortsch. Phys. 57 (2009) 367 [arXiv:0903.2596] [InSPIRE].

[18] H. Liu, J. McGreevy and D. Vegh, Non-Fermi liquids from holography, Phys. Rev. D 83 (2011) 065029 [arXiv:0903.2477] [INSPIRE].

[19] T. Faulkner, H. Liu, J. McGreevy and D. Vegh, Emergent quantum criticality, Fermi surfaces and $A d S_{2}$, Phys. Rev. D 83 (2011) 125002 [arXiv:0907.2694] [InSPIRE].

[20] N. Iizuka, N. Kundu, P. Narayan and S.P. Trivedi, Holographic Fermi and non-Fermi liquids with transitions in dilaton gravity, JHEP 01 (2012) 094 [arXiv:1105.1162] [INSPIRE].

[21] S.A. Hartnoll, D.M. Hofman and D. Vegh, Stellar spectroscopy: fermions and holographic Lifshitz criticality, JHEP 08 (2011) 096 [arXiv:1105.3197] [INSPIRE].

[22] N. Iqbal, H. Liu and M. Mezei, Semi-local quantum liquids, JHEP 04 (2012) 086 [arXiv: 1105.4621] [INSPIRE].

[23] M. Cubrovic, Y. Liu, K. Schalm, Y.-W. Sun and J. Zaanen, Spectral probes of the holographic Fermi groundstate: dialing between the electron star and AdS Dirac hair, Phys. Rev. D 84 (2011) 086002 [arXiv: 1106.1798] [INSPIRE].

[24] U. Gürsoy, V. Jacobs, E. Plauschinn, H. Stoof and S. Vandoren, Holographic models for undoped Weyl semimetals, JHEP 04 (2013) 127 [arXiv: 1209.2593] [INSPIRE].

[25] M. Alishahiha, M.R. Mohammadi Mozaffar and A. Mollabashi, Fermions on Lifshitz Background, Phys. Rev. D 86 (2012) 026002 [arXiv:1201.1764] [INSPIRE].

[26] L.Q. Fang, X.-H. Ge and X.-M. Kuang, Holographic fermions in charged Lifshitz theory, Phys. Rev. D 86 (2012) 105037 [arXiv: 1201.3832] [INSPIRE].

[27] D.T. Son and A.O. Starinets, Minkowski space correlators in AdS/CFT correspondence: Recipe and applications, JHEP 09 (2002) 042 [hep-th/0205051] [INSPIRE].

[28] G.T. Horowitz and M.M. Roberts, Zero temperature limit of holographic superconductors, JHEP 11 (2009) 015 [arXiv:0908.3677] [INSPIRE].

[29] P. Basu, Energy scales in a holographic black hole and conductivity at finite momentum, Can. J. Phys. 89 (2011) 271 [arXiv:0911.5082] [inSPIRE].

[30] C. Keeler, G. Knodel, J.T. Liu and K. Sun, Universal features of Lifshitz Green's functions from holography, JHEP 08 (2015) 057 [arXiv: 1505.07830] [INSPIRE].

[31] N. Iqbal and H. Liu, Universality of the hydrodynamic limit in AdS/CFT and the membrane paradigm, Phys. Rev. D 79 (2009) 025023 [arXiv:0809.3808] [INSPIRE]. 